\title{
Progress of the MINOS experiment
}

\author{
Karol Lang*† \\ The University of Texas, Department of Physics, 1 University Station C1600, Austin, \\ TX 78712-0264, USA \\ E-mail: 'ilang@mail.hep.utexas.edu'
}

ABstract: The MINOS experiment has been designed to study long baseline muon neutrino oscillations in the $\Delta m^{2}$ range of a few $\mathrm{meV}^{2}$. The low-cost magnetized ironscintillator tracking calorimeters, 1 kton at Fermilab and 5.4 kton in the Soudan Mine 735$\mathrm{km}$ away, use industrially extruded scintillator strips with wavelength-shifting and clear fibers coupled to multi-anode photomultipliers. The far detector is now fully completed and commenced collecting cosmic ray data. The neutrino beamline and the near detector will be finished by the end of 2004. In addition, a special calibration detector was also constructed and exposed to the test beams at CERN. MINOS is now emerging as the next generation neutrino oscillation experiment which will determine more precisely the neutrino mixing parameters.

KEYWORDS: neutrino oscilations; neutrino mass; lepton mixing; atmospheric neutrinos; KeY ORDS. neutrino oscilations; neutrino mass, lepton mixing; atmospheric neutrinos; upward going muons; extruded scintillator; multi-anode PMT

\section{Introduction}

The phenomenon of neutrino oscillations provides the most consistent explanation of observations made by experiments with atmospheric, solar, reactor, and recently also accelerator neutrinos. A wave of results generated over the last few years by Super-Kamiokande [2],

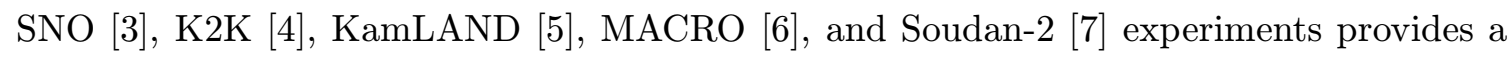
strong evidence of a non-zero mass of neutrinos. It is now conventional to interpret all these studies by picturing atmospheric $\mu$-type neutrinos dominantly oscillating into the $\tau$ type neutrinos, and the solar electron-type neutrinos transforming into $\mu$-type and $\tau$-type neutrinos, with the total flux of neutrinos reaching the earth consistent with that expected from sun [i] or cosmic ray models [i] flavor number is explicitly violated.

${ }^{*}$ Speaker.

${ }^{\dagger}$ Presented on behalf of the MINOS Collaboration [1] [1] 
There is little doubt that these fundamentally new phenomena extend the horizons of physics. Only more studies with higher precision will help to understand them. A new experiment MINOS will make significant new contributions in exploring these phenomena.

\section{The Physics Program of MINOS}

The main goal of MINOS is to conduct a two-detector high-statistics long baseline study of neutrino oscillations $\left[\begin{array}{l}1 \\ 1\end{array} \overline{0}\right]$. MINOS will employ two magnetized toroidal tracking calorimeters, 1 kton at Fermilab and 5.4 kton in the Soudan Mine 735-km away. The experiment will use a well-controlled accelerator beam from the recently completed Main Injector at Fermilab to cover an "atmospheric" range of $\Delta \mathrm{m}^{2}$ and $\sin ^{2}(2 \theta)$ neutrino mixing parameters. The most important physics goals of MINOS could be summarized as follows:

1. Decisive low-systematics observation of the $\nu_{\mu} \rightarrow \nu_{\tau}$ transition and verification of its dominating role in the "atmospheric" $\Delta \mathrm{m}^{2}$ region. This will be detected through the measurement of the disappearance of the charged current (CC) events without loss of neutral current (NC) events, as projected from the near detector to the far detector.

2. Measurements of $\Delta \mathrm{m}^{2}$ and $\sin ^{2}(2 \theta)$ mixing parameters with about $10 \%$ level of accuracy. This will be done by measuring spectral and rate changes of the $\mathrm{CC}$ neutrino interactions in the far as compared to the near detector, as shown in Figures

3. Measurement (or improvement of existing limits) of the $\nu_{\mu} \rightarrow \nu_{e}$ transition for "atmospheric" $\Delta \mathrm{m}^{2}$. This goal will be fulfilled through statistical appearance of electron neutrino interactions (i.e., an excess of this type of interactions in the far detector as projected from the near detector, which will measure the exact $\nu_{e}$ contamination level).

4. Measurement (or improvement of existing limits) of the $\nu_{\mu} \rightarrow \nu_{\text {sterile }}$ transition. This would be signified by simultaneous disappearance of the CC and NC events.

5. Test the CPT symmetry in the atmospheric neutrino interactions through independent detection of the sign of the charge of the $\mathrm{CC}$ muons [i] $\left.\mathrm{i}_{1}\right]$.

6. Test more exotic hypotheses for neutrino transitions, such as neutrino decay [1] $\left.{ }_{2}^{1}\right]$,

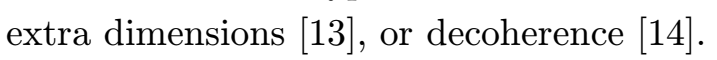

The neutrino beam line NuMI has been designed as a reasonably flexible facility capable of setting the desired neutrino momentum peak between 3 to $18 \mathrm{GeV} / \mathrm{c}$. This has proved valuable over the longer than originally anticipated time scale for the start of the experiment. Since currently favored $\Delta \mathrm{m}^{2}$ for atmospheric neutrinos is around $1-2 \times 10^{-3} \mathrm{eV}^{2}$ MINOS will run primarily with the low energy beam which provides the best precision in this $\Delta \mathrm{m}^{2}$ range, as illustrated in Figure $i_{-}^{2}$ The expected number of reconstructed $\nu_{\mu} \mathrm{CC}$ events is $2,750,8,940$, and 19,940 for low, medium, and high energy beam setting, respectively, per $5 \times 10^{20}$ protons on target. The near detector will reconstruct about $10^{3}$ more events. 

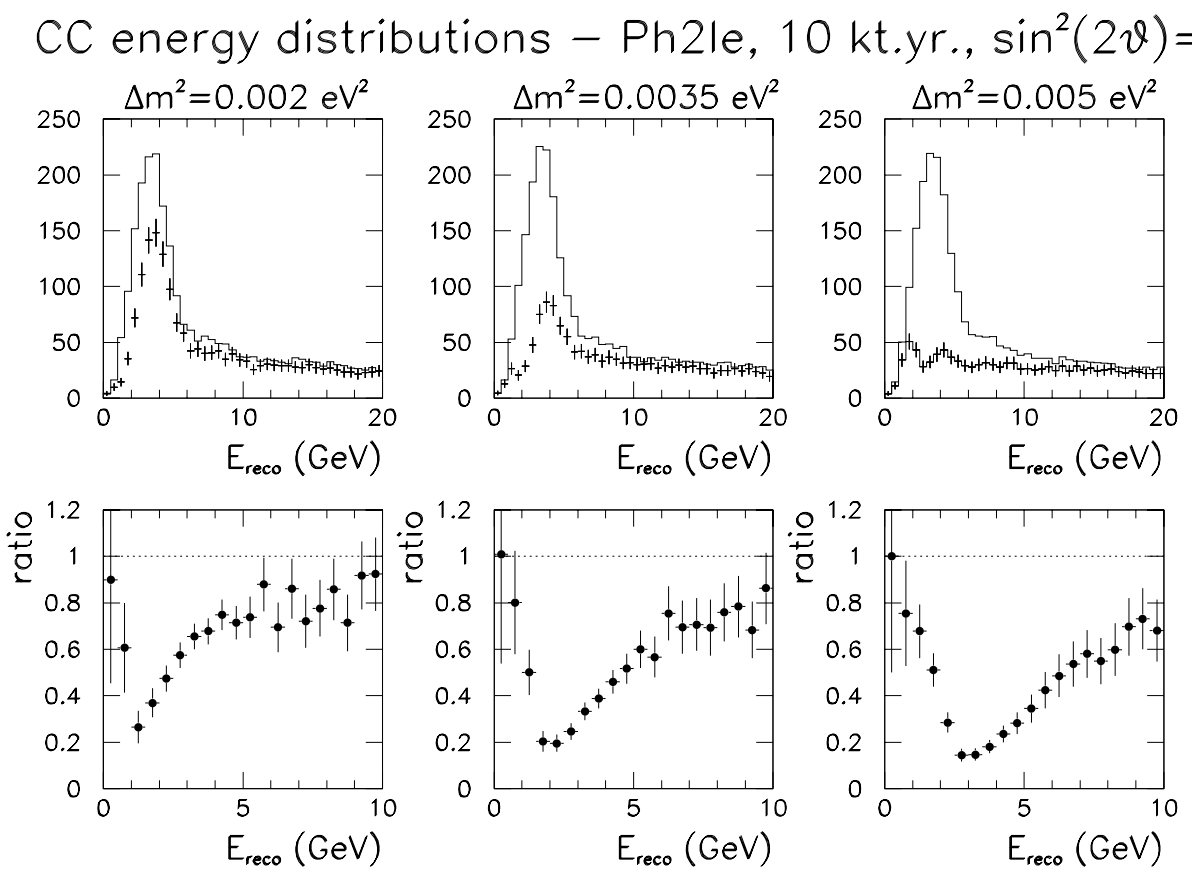

Figure 1: The top plots show the reconstructed energy distributions with and without oscillations for low energy NuMI beam. All plots assume $\sin ^{2}(2 \theta)=0.9$. The solid histograms are the unoscillated distributions and the points with error bars are the oscillated distributions. Statistical fluctuations are suppressed. The bottom plots show the ratio of these two distributions. Contamination of mis-identified neutral current events is included and the true neutrino energies are smeared by resolution functions.

The main signatures for detecting $\nu_{\mu}$ oscillations will be the ratio of the number of charged current $\nu_{\mu}$ events to the number of neutral current events in the two detectors. Also, the rate of $\mathrm{CC}$ events in the far detector, as predicted by the $\mathrm{CC}$ rate in the near detector, and the shape of the energy spectra of the CC events will provide a strong discrimination for $\nu_{\mu}$ disappearance. From the CHOOZ results, albeit for electron anti-neutrinos [i] 1 픔] and assuming the CPT invariance, the $\nu_{\mu} \rightarrow \nu_{e}$ oscillation mode is sub-dominant and indicates a small value of the corresponding mixing angle $\theta_{13}$ of the conventional 3-neutrino mixing matrix [i] $\left[\begin{array}{l}1 \\ \hat{6}\end{array}\right]$. MINOS will be able to detect and statistically measure the $\nu_{e} \mathrm{CC}$ interactions by topological and pulse-height recognition of electromagnetic showers. The intrinsic beam $\nu_{e}$ background is expected at the level of $0.6 \%$ (for low energy beam) to $2 \%$ (for high energy beam). The most serious background in this appearance channel is due to the electromagnetic component (of mostly $\pi^{o}$ 's) of the hadronic shower. Some NC events with fluctuations exhibiting large electromagnetic energy could mimic $\mathrm{CC} \nu_{e}$ interactions. Since both backgrounds, the intrinsic beam and the NC, will be measured in the near detector, the sensitivity of the far detector to $\nu_{\mathrm{e}}$ appearance will be limited by the statistical fluctuation of the number of observed events as compared to the number of events predicted from the near detector. MINOS is expected to improve the current CHOOZ limit by about a factor of $2-3$ at $\Delta m_{13}^{2} \approx 0.003 \mathrm{eV}^{2}$ and will do it initially for neutrinos rather than anti- 

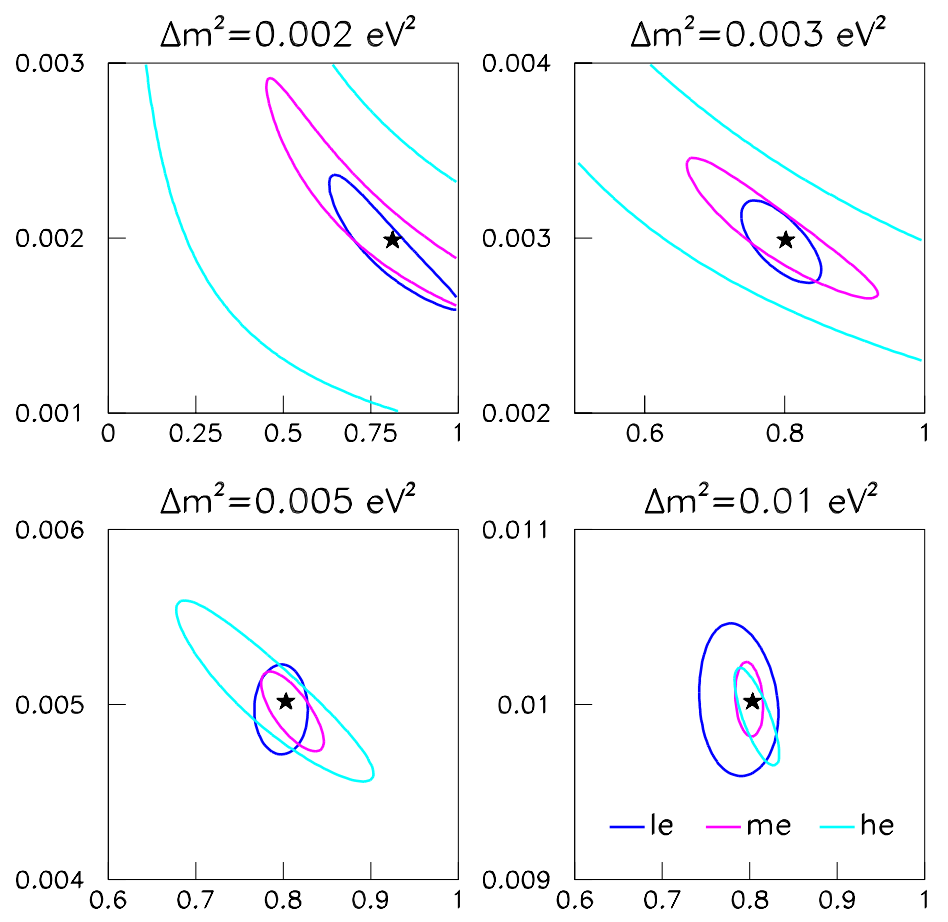

Figure 2: Precision of measurements of neutrino mixing parameters by MINOS. Shown are 1- $\sigma$ contours in $\Delta \mathrm{m}^{2}$ (vertical) and $\sin ^{2}(2 \theta)$ (horizontal) for four different values of true $\Delta \mathrm{m}^{2}$ and three different NuMI beam energies. The value of $\sin ^{2}(2 \theta)$ was taken to be 0.8 for these simulations. The symbols le, me, and he refer to low, medium and high beam energy settings, respectively.

neutrinos. In addition, if oscillated $\nu_{e}$ 's are detected then MINOS could potentially answer the question of neutrino mass hierarchy through matter effects by adding measurements with anti-neutrinos, albeit not with precision of some dedicated experiments planned for the future [1] $\left.\overline{1}_{1} \bar{z}_{1}, \underline{1}, \overline{1}_{1}\right]$.

In summary, several oscillation signatures and optimized neutrino beams will provide MINOS with a variety of control tools for understanding and minimizing measurement uncertainties and to determine neutrino mixing parameters.

\section{The NuMI beam}

A new neutrino beamline NuMI is about $1 \mathrm{~km}$ long and declines toward the Soudan Mine in Minnesota at a $58 \mathrm{mrad}$ angle. It will use the Main Injector proton beam of energy $120 \mathrm{GeV}$ and intensity of $2.5-4 \times 10^{13}$ protons on target per spill. The Main Injector will receive up to 6 batches from the Booster accelerator, of which 5 batches will be used for NuMI. The primary beam will be extracted from the Main Injector in a $9.8 \mu$ s-long single-turn extraction every $1.9 \mathrm{~s}$ onto a graphite target of $6.4 \times 18 \times 940 \mathrm{~mm}^{3}$ segmented longitudinally into 47 fins. The target will be water cooled and is designed to withstand 
up to $4 \times 10^{13}$ protons per spill. A prototype target was successfully tested in the Main Injector at peak energy densities exceeding those expected for MINOS.

Particles produced in the target will be focused by two magnetic "horns", which generate strong focusing toroidal magnetic field when pulsed with the $200 \mathrm{kA}$ peak-current. Sign and momentum selected secondaries enter then the $675 \mathrm{~m}$ long and $2 \mathrm{~m}$ diameter decay pipe. The two-horn system will produce a wide-band neutrino beam. The energy of the beam can be tuned by the relative placement of the two horns and the target, thus focusing pions and kaons of a particular momentum band. MINOS will be able to tune the energy spectrum of the $\nu_{\mu}$ neutrino beam from about 3 to $18 \mathrm{GeV}$ by fixing the first horn and relocating the downstream horn.As mentioned earlier, the low energy configuration is foreseen for the initial main running period.

At the end of the decay volume the beamline will be instrumented with ionization chambers to monitor the secondary beam. There will be one hadron monitoring station, located just upstream of the beam absorber (a water-cooled aluminum core, iron, and concrete block structure), and three muon monitoring stations, located in three caves downstream of the absorber (with $0 \mathrm{~m}, 8 \mathrm{~m}$, and $12 \mathrm{~m}$ rock thickness to the absorber). The pointing accuracy of the muon stations will allow to align the neutrino beam direction to about $50 \mu$ radians in one spill. Tests conducted with the prototype chambers indicate that the expected particle flux of up to $10^{9} / \mathrm{cm}^{2} /$ spill should pose no serious operational problems though the lieftime expectancy of the hadron monitor is short due to high radiation dose expected. These main features and a number of target, beam transport, and focusing elements will be optimized to assure that the beam's energy spectrum, its divergence, and composition will be well understood and controlled.

\section{The near and far detectors}

The smaller mass, 1 kton, "near" detector will be placed 1,040 m downstream of the target, or $300 \mathrm{~m}$ downstream of the end of the decay pipe, and about $100 \mathrm{~m}$ underground. The near detector will assure precise determination of the neutrino beam characteristics such as the energy spectrum, the rate of neutral to charged current events, and the $\nu_{\mathrm{e}}$ beam contamination. The entire detector will provide continuous beam monitoring while only the small region within $25 \mathrm{~cm}$ radius around the beam axis will be used for "near-far" neutrino oscillation analysis. The large mass, $5.4 \mathrm{kton}$, "far" detector is placed in the Soudan Mine $735 \mathrm{~km}$ away from the near detector and $713 \mathrm{~m}$ underground. The role of this detector will be to measure the rate and energy of the $\nu_{\mu}$ neutral-current and charged current interactions and identify statistically a fraction of $\nu_{\mathrm{e}}$, and for higher energies also $\nu_{\tau}$ events. Both detectors will have an identical segmentation, but will be differently optimized to minimize biases in reconstruction of neutrino events occurring at the two locations with a difference in the neutrino flux of more than $10^{5}$.

The far detector consists of 485 iron-scintillator planes assembled in two super-modules, each an $8 \mathrm{~m}$-diameter octagonal toroid. Each $2.54 \mathrm{~cm}$-thick steel plane (plug-welded out of 8 plates in two layers) supports a $1 \mathrm{~cm}$-thick and $4.1 \mathrm{~cm}$-wide scintillator strips grouped into 20- and 28-strip wide light-tight modules. The scintillator strips have been made 
in the industrial extrusion process using STYRON 663W polystyrene, manufactured by The Dow Chemical Company, doped with $1 \%$ of PPO and $0.03 \%$ of POPOP. In addition, a $0.25 \mathrm{~mm}$-thick reflective layer, obtained by adding $12.5 \%$ of $\mathrm{Ti}_{2}$ to polystyrene and co-extruding it with the scintillator, surrounds each strip, except along a $1.4 \mathrm{~mm}$-wide and $2.0 \mathrm{~mm}$-deep groove also co-extruded for embedding a light-collecting $1.2 \mathrm{~mm}$-diameter wavelength-shifting (WLS) fiber. The WLS fibers are a J-type Y11 multi-clad PMMA, nonS, made by Kuraray, and doped with K27 dye at 175 ppm (maximum intensity emission at $520 \mathrm{~nm}$ ). The fibers are optically coupled to the scintillator strips with EPON 815C epoxy manufactured by Shell. The light attenuation in the WLS fibers is well modeled by a double-exponential fall-off with $\lambda_{1} \approx 0.7 \mathrm{~m}$ and $\lambda_{2} \approx 3.9 \mathrm{~m}$. Signals from WLS fibers are transported by clear polystyrene fibers, made by Kuraray, with the attenuation length $\lambda \approx 13 \mathrm{~m}$.

In the far detector the fibers are read out from both ends. They are grouped (multiplexed) in a fiber-multiplexing box into sets of 8 fibers from strips spaced apart by about $1 \mathrm{~m}$ in a scintillator plane. Each 8-fiber bundle is terminated in a "cookie" which is directly coupled to a 16-pixel R5900-M16 photomultiplier (PMT) manufactured by Hamamatsu Photonics. Each PMT reads out 128 fibers; one end of each scintillator plane needs 24 pixels, or 1.5 PMT. Altogether, MINOS uses about 1,550 of the 16-anode PMTs. By grouping fibers differently at the opposite ends an unambiguous pattern recognition can be achieved. A sum of light from two ends is fairly flat across the entire detector and at a level of 8-12 photoelectrons. The near detector will be a $3.8 \mathrm{~m}$ by $4.8 \mathrm{~m}$ "squeezed" octagonal toroid made of solid $2.54 \mathrm{~cm}$ iron plates and interspersed with similar scintillator modules. There will be two main longitudinal sections in the near detector. The target/hadronshower calorimeter will have scintillator modules covering about one half of the transverse area in 96 planes and a full-area coverage in 24 planes (every 4th plane). To track muons the downstream section (164 iron planes) will have every 5th gap (33 planes) instrumented with the full-area scintillator coverage. Fibers will be read out individually from one end by about 215 64-anode Hamamatsu PMTs. The opposite ends will be reflectively terminated to boost an average light yield to a similar level as in the far detector.

Both detectors will be magnetized (using coils through a hole in the middle of each plane) to a field of about $1.5 \mathrm{~T} 2 \mathrm{~m}$ away from the coil. The front-end electronics will be different for the two calorimeters to optimize the cost and efficiency of event reconstruction and deal with multiple events per spill in the near detector. In the far detector the front-end electronics is based on the VA chip from IDE Inc., and in the near detector it is based on the modified QIE chip designed at Fermilab and used there previously. Simulations, as well as test beam results presented below, show that the response of these tracking calorimeters will be $55 \% / \sqrt{\mathrm{E}}$ and $23 \% / \sqrt{\mathrm{E}}$ for hadronic and electromagnetic showers, respectively. Both detectors will be calibrated using cosmic ray muons, light injection system, and using results form a test beam exposure of the special calibration module discussed below.

\section{Calibration detector}

One of the biggest technical challenges before MINOS will be to determine the energy scale 
between the two distant detectors located deep underground to within $2 \%$ relative between the near and far locations, and 5\% absolutely in each location. A fundamental component of understanding the detector response to muons, hadrons, and electrons is a series of test beam runs which employ a special MINOS calibration detector (CalDet).

CalDet is essentially a non-magnetized mini-MINOS detector, sufficiently large to contain hadronic and electromagnetic showers with energy of several GeV. It consists of 60 $1 \times 1 \mathrm{~m}^{2}$ MINOS iron-scintillator planes, and is equipped with phototubes and electronics from the far and near detectors. Each end of the 1440 scintillator strips is read out at both ends. The wavelength-shifting fibers are lengthen to simulate a large size of the far detector. Measurements of the response of CalDet to muons, electrons, and hadrons will provide the transfer of energy scale from cosmic muons to the energy of neutrino interactions. In MINOS, the cosmic ray muons will be used for a relative calibration of each scintillator strip, while a light injection system will provide a short-term drift corrections. In addition, both the near and the far front-end electronics systems will have the capability of self-calibration using charge injection circuits.

The beam exposure of CalDet at the PS at CERN, where suitable test beams are available, have now been completed. For the exposure in 2001 the entire CalDet was instrumented with the far detector phototubes and electronics. In 2002, the test continued at higher energies and also some initial tests of the near detector electronics were conducted by instrumenting one side of 6 planes with the near detector PMTs and prototype frontend electronics. In the 2003 exposure modules were initially read out simultaneously from both fiber-ends with two types of front-end electronics systems, and later the detecor was configured to mimic the near detector arrangement. This three-year exposure program provides a full response matrix to all types of particles, and allows a direct comparison of response and calibration between the two detectors.

CalDet was run in the T11 and T7 test beamlines of the CERN PS complex. Full analysis of the collected data is under way [i] $\left[\begin{array}{l}1 \\ 9\end{array}\right]$. The T11 test beam provided particles with a maximum momentum of $3.6 \mathrm{GeV} / \mathrm{c}$, and a momentum spread of $3 \%$, while $\mathrm{T} 7$ can be tuned from 1 to $10 \mathrm{GeV} / \mathrm{c}$. The beam consisted of charge-selected pions, protons, electrons, and muons. For particle identification the beamlines were equipped with Cerenkov counters and a pair of time-of-flight scintillators which also served as the trigger counters.

The energy of electrons can be expressed in terms of the muon energy unit (MEU), defined as the energy deposited by a normally incident, through-going muon read out from both strip ends. On average, electrons deposit in CalDet about 60 MEU's per GeV. Fits to these distributions show that the electromagnetic energy resolution is $23 \% / \sqrt{E}$, thus in agreement with expectations. Figure $\underline{\beta}_{1}$ shows preliminary distribution of the total energy deposited by identified pions and protons. While at this preliminary state of analysis there is still some muon contamination in both samples, these plots clearly indicate that, as expected, pions induce more activity (i.e., display more visible energy) in the detector than protons with the same momentum. Fits to these distributions show that the hadronic energy resolution is at the expected level of $55 \% / \sqrt{E}$. Finally, as preliminary results shown in Figure isicindicate, the response of the two types of front-end electronics to the same events is very similar and, as expected, does not introduce any reconstruction biases. 

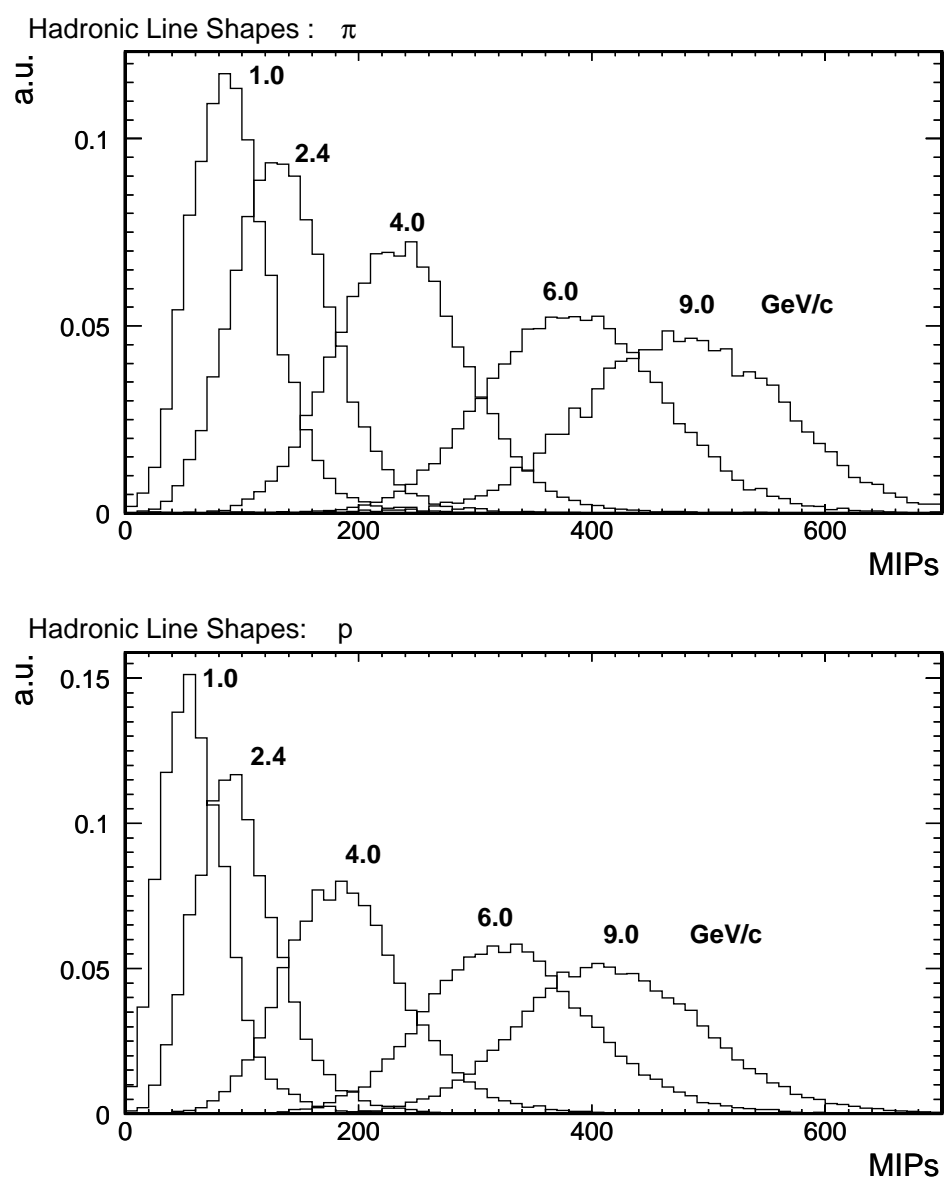

Figure 3: Preliminary results from CalDet. Energy distributions in CalDet for identified pions (top) and protons (bottom) for beam momenta in the CERN PS T7 and T11 beamlines set from -1 to $-9 \mathrm{GeV}$. The energy is expressed in muon energy units, defined as the energy deposited by a normally-incident, through-going muon read out from both strip ends. Distributions are normalized to fit in one histogram. Fits to these distributions give the hadronic energy resolution of $55 \% / \sqrt{E}$.

The analysis of the CalDet data continues and will include a thorough study of energy response, topology and Monte Carlo modeling of the detector and the test beam data. These will be very important ingredients in reconstruction the energy and topology of the neutrino events in the nominal MINOS detectors and in identifying neutral and charged current as well as electron-shower events.

\section{MINOS Observatory}

The far MINOS detector is located deep underground and can naturally serve as a cosmic ray observatory. The two super-modules are now fully completed and energized and MINOS Observatory commenced its full operation in late summer 2003. This is the first large underground detector which is magnetized and thus capable of measuring momenta and 
Total MIP, T7 2003 (Run 70771)

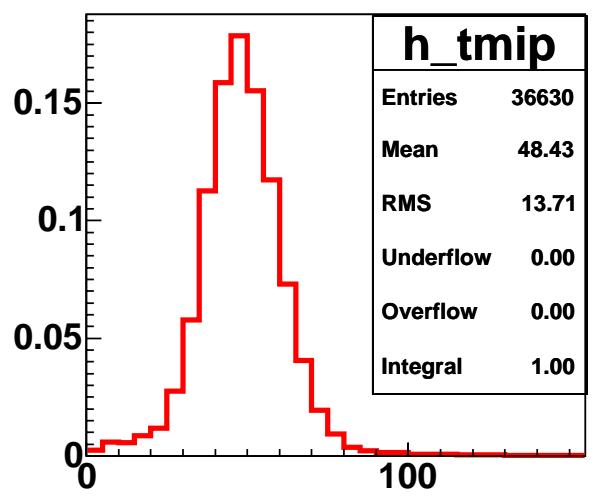

P. Vahle,2003/09/15

Total MIP, Far

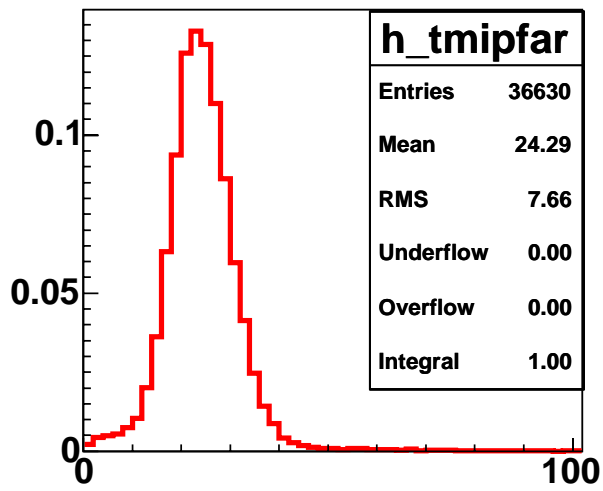

Total MIP, Near vs. Far

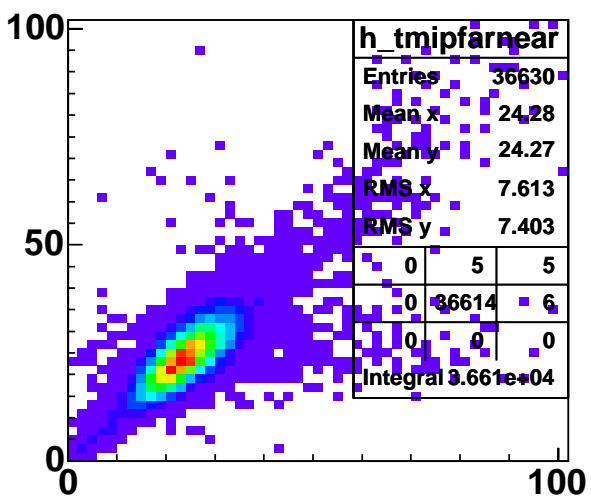

Total MIP, Near

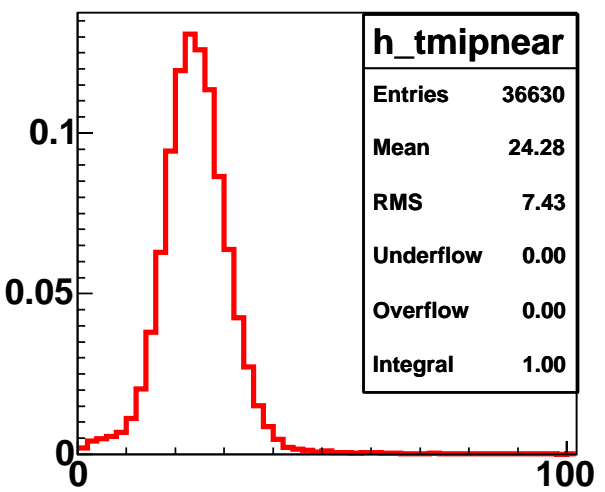

Figure 4: Preliminary results from CalDet. Event-by-event comparison of the response of the near and far front-end electronics. Top right is the scatter plot of energy (expressed in MIPs or MEUs, see text) reconstructed on the left side using the near detector electronics versus that of the right side using the far detector electronics. The other plots are corresponding projections.

the sign of the cosmic ray muons. This unique feature will allow MINOS to conduct the first CPT check of the atmospheric neutrino oscillations [i]1] MINOS will study the two main classes of neutrino events: those with an interaction vertex contained within the fiducial volume of the detector, and those which interact in the rock in the vicinity of the cavern and produce muons which are registered by the detector. Table number of cosmic ray events expected in the far detector. The MINOS observatory will be able to fully reconstruct high energy neutrino interactions and measure muon momenta up to about $70 \mathrm{GeV}$ (or higher for longer events). In addition, using calorimetric response, MINOS will be able to search for very high energy muons $(>10 \mathrm{TeV})$.

The rate of atmospheric muons in MINOS is $10^{5}$ higher than the rate of cosmic ray neutrino interactions in the detector. Because the acceptance of the detector is optimized for the beam neutrinos from Fermilab, tracks which are perpendicular to the main axis of the detector have limited detection acceptance. Cosmic muons, whose typical energy 


\begin{tabular}{lcccc}
\hline & $\nu_{\mu}$ & $\bar{\nu}_{\mu}$ & $\nu_{\mu}$ & $\bar{\nu}_{\mu}$ \\
& $\Delta \mathrm{m}^{2}=0$ & $\Delta \mathrm{m}^{2}=0$ & $\Delta \mathrm{m}^{2}=0.003$ & $\Delta \mathrm{m}^{2}=0.003$ \\
Contained vertex with a muon & 620 & 400 & 440 & 260 \\
Upgoing muons & 280 & 120 & 280 & 120 \\
\hline
\end{tabular}

Table 1: Cosmic ray physics potential of MINOS. The expected number of reconstructed cosmic ray events in the far detector. The numbers are shown for the oscillation scenario $\left(\Delta \mathrm{m}^{2}=0.003\right)$ and no oscillation scenario $\left(\Delta \mathrm{m}^{2}=0\right)$ hypotheses and are normalized to a 5 -year exposure.

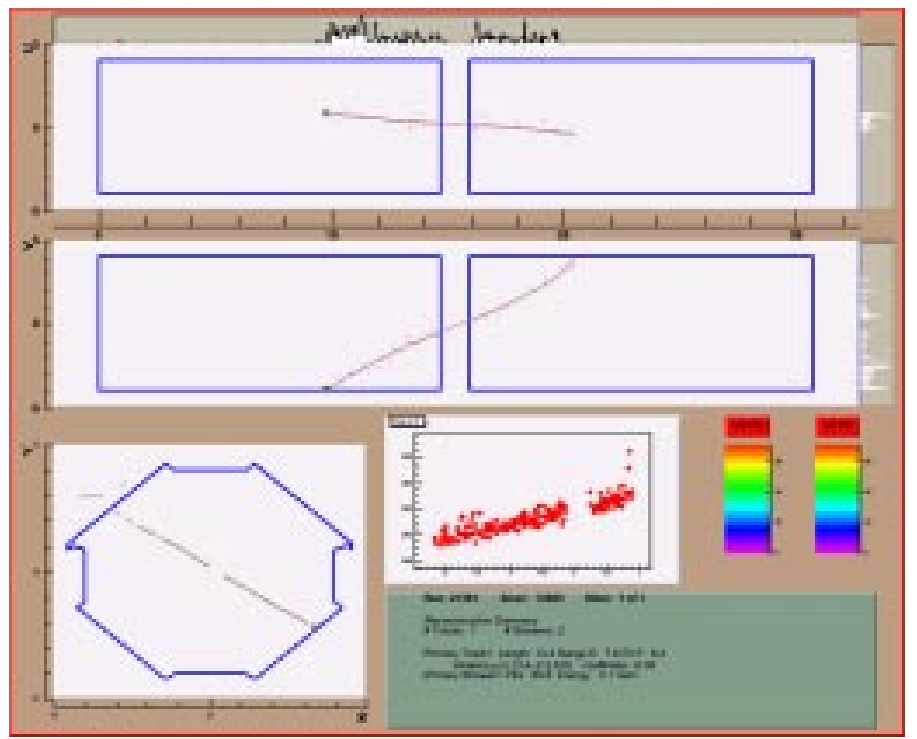

Figure 5: Preliminary results from MINOS Observatory. Display of the event: Run 21361, Snarl 12693. An example of an upward-going muon event. Top four plots show hits in the scintillator planes in Super Module 1 and 2. Bottom left shows the face-on transverse distribution of hits. Bottom right shows the times of hits as a function of the vertical distance from the center of the detector. The positive correlation strongly indicates an upward going track.

and rate in the MINOS cavern is $200 \mathrm{GeV}$ and $0.22 \mathrm{~Hz}$, respectively, can enter undetected through an iron plate and interact in the detector thus producing an apparent contained event. This lack of hermeticity for side entrance is a serious source of possible backgrounds for the contained events sample. Upward-going muon events are less vulnerable to such backgrounds, as it is illustrated in Figure 系 which shows a candidate for an upward-going muon event. An effective tool to suppress such background is a veto shield which surrounds the detector. A number of upward-going candidates has been identified and their direction can be compared to upward-going muons, as illustrated in Figure $\overline{\mathbf{T}}_{1}$. The veto shield also enables a search for contained neutrino events. Figure $\overline{\mathbf{6}}$ shows the first strong candidate for a contained neutrino event. 


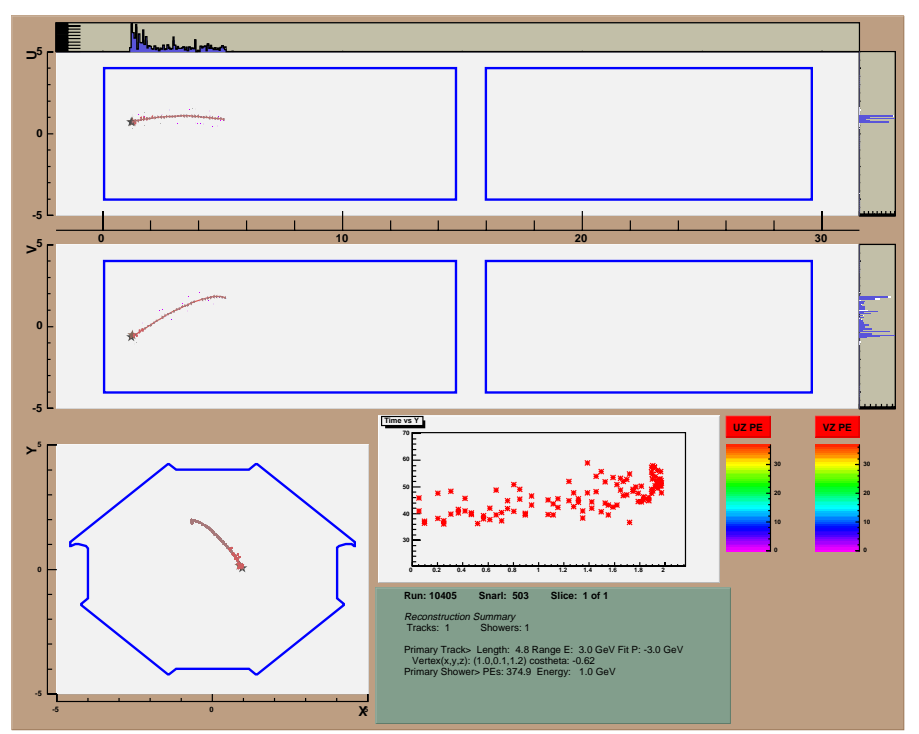

Figure 6: Preliminary results from MINOS Observatory. Display of the event: Run 10405, Snarl 503. One of the first candidate events for a contained neutrino interaction. Top four plots show hits in the scintillator planes in Super Module 1 and 2. Bottom left shows the face-on transverse distribution of hits. Bottom right shows the times of hits as a function of the vertical distance from the center of the detector. No activity in the veto shield was registered.

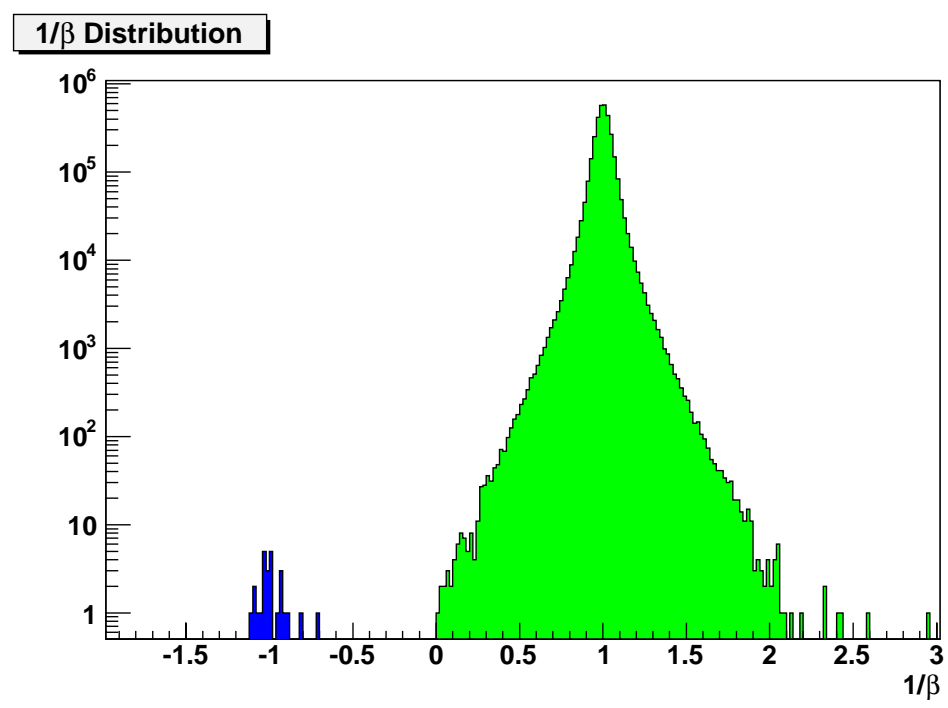

Figure 7: Preliminary distribution of the inverted normalized speed of reconstructed muons. Downward-going muons occupy +1 region while upward-going muons cluster around -1 .

\section{Current Status of the Project}

The progress of NuMI and MINOS has been on budget and schedule since the project was re-baselined in September 2001. The current cost and schedule, extended largely due to delays in the civil construction of the underground facilities, have been firmly kept on track ever since. The tunnel is completed and instrumented with the decay pipe and its 
concrete shielding. Construction of the surface building and outfitting of the near detector hall are also close to completion. It is now realistic to projects the first protons on target in December 2004 and observation of first beam neutrinos in both detectors shortly after.

The near detector planes have all been pre-assembled at Fermilab and are ready for their final installation in the near detector hall. This will commence in January 2004. The near detector will then be installed within the following 6 months.

The NuMI beamline is now fully designed, but its construction remains on the critical path. The beam effort is now focusing on understanding the performance of Booster and the Main Injector accelerator complex. Measurements are under way to understand several design parameters, including momentum dispersion and beam emittance. Improved Booster and better understanding of Main Injector are vital for providing sufficient number of protons on NuMI target to keep the program on the proposed path. MINOS is now looking into the near future with great optimism.

\section{References}

[1] The MINOS Collaboration includes research groups from Argonne National Laboratory, University of Athens, Brookhaven National Laboratory, California Institute of Technology, University of Cambridge, University of Campinas, College de France, Fermi National Accelerator Laboratory, Harvard University, Illinois Institute of Technology, Indiana University, ITEP Moscow, Lebedev Institute, Lawrence Livermore National Laboratory, University College London, Macalester College, University of Minnesota, University of Minnesota-Duluth, Northwestern University, University of Oxford, University of Pittsburgh, IHEP Protvino, Rutherford Appleton Laboratory, University of Sao Paolo, University of South Carolina Stanford University, University of Sussex, Texas A\&M University, University of Texas at Austin, Tufts University, Western Washington University, and Universisty of Wisconsin.

[2] S. Fukuda et al. [Super-Kamiokande Collaboration], Phys. Rev. Lett. 85, 3999 (2000)

S. Fukuda et al. [Super-Kamiokande Collaboration], Phys. Lett. B 539, 179 (2002)

[3] Q. R. Ahmad et al. [SNO Collaboration], Phys. Rev. Lett. 89, 011301 (2002)

S. N. Ahmed et al. [SNO Collaboration], arXiv:nucl-ex/0309004.

[4] M. H. Ahn et al. [K2K Collaboration], Phys. Rev. Lett. 90, 041801 (2003)

[5] K. Eguchi et al. [KamLAND Collaboration], Phys. Rev. Lett. 90, 021802 (2003)

[6] M. Ambrosio et al. [MACRO Collaboration], Phys. Lett. B 566, 35 (2003)

[7] M. Sanchez et al. [Soudan 2 Collaboration], arXiv:hep-ex/0307069.

W. W. M. Allison et al. [Soudan-2 Collaboration], Phys. Lett. B 449, 137 (1999)

[8] J. N. Bahcall and M.H. Pinsonneault, and S. Basu, Astrophys.J. 555:990-1012,2001;

J. N. Bahcall, S. Basu and M.H. Pinsonneault, Phys.Lett. B433:1-8,1998.

[9] T.K. Gaisser and M. Honda, hep-ph/0203272;

M. Honda, T. Kajita, K. Kasahara and S. Midorikawa, Phys.Rev. D64:053011,2001;

M. Honda, T. Kajita, K. Kasahara and S. Midorikawa, Phys.Rev. D52:4985-5005,1995;

T.K. Gaisser and T. Stanev, Eur.Phys.J. C15:150-156,2000.

[10] Fermilab Proposal P875, February, 1995; The MINOS Detectors, Technical Design Report, Fermilab, NuMI-L-337, October, 1998. 
[11] G. Barenboim, L. Borissov, and J. Lykken, hep-ph/0212116, FERMILAB-Pub-02/329-T.

[12] Vernon D. Barger at al, Phys. Lett. B462:109-114,1999;

Vernon D. Barger at al, Phys. Rev. Lett. 82:2640-2643,1999.

[13] H. Davoudiasl, P. Langacker, and M. Perelstein, Phys. Rev. D65:105015,2002;

[14] T. Ohlsson, Phys. Lett. B502:159-166,2001;

E. Lisi, A. Marrone, and D. Montanino, Phys. Rev. Lett. 85:1166-1169,2000

[15] M. Apollonio et al., Phys.Lett. B466:415-430,1999.

[16] B. W. Lee, S. Pakvasa, R. Shrock, and H. Sugawara, Phys.Rev.Lett. 38:937,1977;

B. W. Lee and R. Shrock, Phys.Rev. D16:1444,1977;

Z. Maki, M. Nakagawa, S. Sakata, Prog. Theor. Phys. 28:870,1962;

[17] Letter of Intent to build an Off-axis Detector to study $\nu_{\mu} \rightarrow \nu_{e}$ oscillations with the NuMI Neutrino Beam, D. Ayres et al, hep-ex/0210005, October 2, 2002.

[18] The JHF-Kamioka Project, Y. Itow et al, hep-ex/0106019, June 5, 2001.

[19] P. Vahle et al., Prepared for 10th International Conference on Calorimetry in High Energy Physics (CALOR 2002), Pasadena, California, 25-30 Mar 2002, R. Nichol et al., ibid. 